\title{
Diversidad y variación estacional de peces en la cuenca baja del río Nanay, Perú
}

\section{Fishes species diversity and seasonal variation in the lower basin of Nanay River, Peru}

Ericka Correa ${ }^{1}$ y Hernán Ortega ${ }^{1}$

1 Departamento de Ictiología. Museo de Historia Natural, Universidad Nacional Mayor de San Marcos, Av. Arenales 1250, Jesís Marí, Apartado 14-0434 Lima 4, Peru.

Email Ericka Correa:

evanessa.correa@gmail.com

Trabajo presentado a la XVIII Reunión Científica del Instituto de Investigaciones en Ciencias Biológicas Antonio Raimondi, "200 años del nacimiento de Charles Darwin y el 150 aniversario de la publicación de On the Origin of Species by Means of Natural Selection". De 19 al 21 de agosto de 2009 .

Publicado impreso: 20/10/2010 Publicado online: 29/09/2010

\section{Resumen}

El presente trabajo informa sobre la diversidad de peces en la cuenca baja del rio Nanay, tributario del río Amazonas. Esta zona está sometida a la extracción indiscriminada de peces con usos ornamentales y de consumo directo. Utilizando redes de arrastre $(10 \times 2,5 \mathrm{~m}, 5 \mathrm{~mm}$ de luz de malla), se realizaron capturas en época de vaciante (agosto y setiembre de 2007) y creciente (febrero de 2008), en tres lugares del rio Nanay. Se colectaron 1626 individuos, correspondientes a 86 especies, de 23 familias y cinco órdenes. El número de especies vario entre 13 y 21 en época de vaciante y entre 18 y 26 especies en época de creciente. Predominaron los Characiformes, Siluriformes y Perciformes. El 76\% de los individuos con tamaños menores de 10 $\mathrm{cm}$. El $65 \%$ de las especies registradas tienen uso ornamental.

Palabras clave: Peces ornamentales, Amazonía, estacionalidad, pesca, Loreto.

\section{Abstract}

This paper reports on the diversity of fish in the lower basin of the Nanay River, a tributary of the Amazon River. This area is subjected to indiscriminate harvesting of fish for ornamental purposes and for direct consumption. Using trawl nets $(10 \times 2.5 \mathrm{~m}, 5 \mathrm{~mm}$ mesh), we fished in the dry season (August and September 2007) and wet season (February 2008) in three places in the Nanay River. 1626 individuals were collected, corresponding to 86 species of 23 families and five orders. The number of species varied between 13 and 21 in the dry season and between 18 and 26 species during the growing season. Characiformes, Siluriformes and Perciformes were the dominant orders. $76 \%$ of individuals had small sizes. $65 \%$ of the recorded species have ornamental use.

Keywords: Ornamental fish, Amazon, seasonality, fishing, Loreto.

\section{Introducción}

Uno de los principales tributarios del río Amazonas en territorio peruano es el río Nanay, ubicado en el departamento de Loreto, caracterizado por ser un río de agua negra (Roldán 1992, Sioli 1984, Ortega \& Castro 1998), y comprende muchos tipos de microhábitats que albergan gran diversidad de peces importantes para el consumo humano y de uso ornamental, además de presentar en las áreas cercanas (riberas y planicies inundables) con un alto nivel de endemismo (Hidalgo \& Willink 2007).

Entre los problemas que se han observado en relación al río Nanay, se encuentra la extracción indiscriminada de peces ornamentales, alentado por el comercio acuarista existente en la zona y que satisface las grandes demandas provenientes de países de Europa, Norteamérica y Asia. Así, el centro de exportación de peces de acuario en el Perú es Iquitos, donde el comercio ha estado activo desde la década de 1950 (Gerstner et al. 2006). La presión en cuanto a la extracción de peces ornamentales en el río Nanay es elevada, sobretodo en la parte baja (Tello \& Cánepa 1991, Souza et al. 2004, Gerstner et al. 2006).

En el presente trabajo se informa de la composición taxonómica y la estructura comunitaria de peces de la cuenca baja del río Nanay, en épocas de creciente y vaciante; y se actualiza la información de la diversidad de peces del río Nanay, concocimiento que permitirá reforzar las medidas de conservación.

\section{Materiales y métodos}

El área de estudio se localiza en el Departamento de Loreto, provincia de Maynas, cerca a la ciudad de Iquitos. Se colectaron las muestras en la cuenca baja del río Nanay, que comprende desde el caserío de Salvador de Pava hasta su desembocadura en la margen izquierda del Río Amazonas (IIAP, 2002). Se eligieron las localidades Las Camelias, Pampachica, Nina Rumi y Puerto Almendras cercanas a la ciudad de Iquitos (034' $\mathrm{S}$, $73^{\circ} 14^{\prime} \mathrm{W}$, entre 84 y $92 \mathrm{~m}$ altitud), ubicadas en el distrito de San Juan Bautista, las dos últimas con acceso desde la carretera Iquitos-Nauta, a la altura del kilometro siete, al suroeste de la ciudad de Iquitos.

Las colectas fueron realizadas en los lugares frecuentados por los pescadores de peces ornamentales, como son las orillas, palizadas (formada por ramas); árboles sumergidos y vegetación flotante, que se entrelazan en las curvas de los meandros, de los canales de los ríos o en las "tahuampas" (Souza et al., 2004); "gramalotales", comunidades de gramíneas herbáceas robustas compuestas por varias especies que ocupan los bordes de las playas y bancos de arena planos o de suave declive (Kahn et al. 1993). Estos lugares presentan diferentes niveles de presión de extracción de peces ornamentales, según Souza et al. (2004). También se colectó en el hábitat de sustrato arenoso.

En cada localidad se realizaron cinco lances por estación, en época de vaciante, agosto y setiembre de 2007 y creciente, febrero de 2008. Se utilizó una red de arrastre a la orilla de 10 x $2,5 \mathrm{~m}$ y $5 \mathrm{~mm}$ de apertura de malla. Los peces fueron fijados en formol al $10 \%$ y preservados en etanol al $70 \%$ en el campo. Fueron identificados, catalogados y depositados en la colección científica del Museo de Historia Natural de la Universidad Nacional de San Marcos (UNMSM).

Se realizó la descripción de los hábitats, con información de temperatura del aire, temperatura del agua, $\mathrm{pH}$, transparencia, conductividad y oxígeno disuelto.

Se determinó la composición taxonómica y abundancia relativa de la comunidad de peces. Los valores de abundancia relativa, se obtuvieron en base a la presencia y frecuencia de ocurrencia (Ortega, 2007). 
Tabla 1. Descripción de hábitat y parámetros fisicoquímicos de los cuerpos de agua evaluados en la cuenca baja del Río Nanay.

\begin{tabular}{|c|c|c|c|c|c|c|c|c|}
\hline \multirow{2}{*}{ Características } & \multicolumn{4}{|c|}{ Vaciante } & \multicolumn{4}{|c|}{ Creciente } \\
\hline & Pampachica & $\begin{array}{c}\text { Las } \\
\text { Camelias }\end{array}$ & $\begin{array}{c}\text { Puerto } \\
\text { Almendras }\end{array}$ & Ninarumi & Pampachica & Las Camelias & $\begin{array}{c}\text { Puerto } \\
\text { Almendras }\end{array}$ & Ninarumi \\
\hline Cuerpo de agua & Lotico & Lentico & Lotico & Lotico & Lotico & Lotico & Lotico & Lotico \\
\hline Hábitat & Playa arenosa & Laguna & Playa arenosa & Playa arenosa & Gramalotal & $\begin{array}{l}\text { Laguna con } \\
\text { macrofitas }\end{array}$ & Palizada & Gramalotal \\
\hline Velocidad de Corriente & Moderada & Nula & Lenta & Lenta & Lenta & Nula & Nula & Nula \\
\hline Tipo de agua & Negra & Negra & Negra & Negra & Negra & Negra & Negra & Negra \\
\hline Color de agua & Té oscuro & Té oscuro & Té oscuro & Té oscuro & Té oscuro & Té oscuro & Té oscuro & Té oscuro \\
\hline Amplitud de cauce & 80 & 40 & 70 & 60 & 115 & 20 & 110 & 100 \\
\hline Área de muestreo $\left(\mathrm{m}^{2}\right)$ & 400 & 200 & 400 & 400 & 300 & 200 & 200 & 300 \\
\hline Profundidad (m) & 1,1 & 0,8 & 1,5 & 1,5 & 1,5 & 1,55 & 1,55 & 1,35 \\
\hline Substrato & Arenoso & $\begin{array}{l}\text { Areno- } \\
\text { fangoso }\end{array}$ & Arenoso & Arenoso & Arenoso & $\begin{array}{l}\text { Areno- } \\
\text { fangoso }\end{array}$ & $\begin{array}{l}\text { Areno- } \\
\text { fangoso }\end{array}$ & $\begin{array}{l}\text { Areno- } \\
\text { fangoso }\end{array}$ \\
\hline Vegetación & Arbustiva & $\begin{array}{l}\text { Herbácea- } \\
\text { arbustiva }\end{array}$ & $\begin{array}{c}\text { Herbácea- } \\
\text { arbustiva }\end{array}$ & Herbácea & Herbácea & Herbácea & $\begin{array}{c}\text { Herbácea- } \\
\text { arbustiva }\end{array}$ & Herbácea \\
\hline T. del aire $\left({ }^{\circ} \mathrm{C}\right)$ & 29 & 31 & 32 & 31 & 34,5 & 34 & 32 & 32 \\
\hline T. del agua $\left({ }^{\circ} \mathrm{C}\right)$ & 27,5 & 28,5 & 29,4 & 28,5 & 28,75 & 30,5 & 26,8 & 28 \\
\hline $\mathrm{pH}$ & 6,98 & 7,06 & 6,53 & 6,74 & 6,4 & 6,54 & 7,03 & 8,27 \\
\hline Conductividad $(\mu \mathrm{S} / \mathrm{cm})$ & 7,65 & 8 & 7,03 & 8,2 & 9,1 & 8,16 & 8,03 & 11,3 \\
\hline Transparencia $(\mathrm{cm})$ & 15 & 14 & 16 & 16 & 15 & 15 & 15 & 15 \\
\hline Oxígeno disuelto (ppm) & 4 & 3,82 & 3,8 & 3,86 & 3,7 & 3,58 & 3,63 & 3,5 \\
\hline
\end{tabular}

Se realizó un análisis de similaridad comparando los lugares de estudio y las épocas de muestreo (Manly, 1985). Se uso las la Distancia Euclidiana entre los casos, y se elaboro un dendrograma.

El índice de abundancia fue estimado de acuerdo al esfuerzo realizado, es decir por lances y estaciones, teniendo en cuenta que fueron 5 lances por estación ( $\mathrm{n}^{\mathrm{o}}$ de individuos/lance) y 4 estaciones por épocas ( $n^{\circ}$ de individuos/estación) (Ortega 2007). Se compararon también las abundancias relativas entre estaciones, hábitat, épocas y zona de estudio, considerando las proporciones obtenidas en cada orden y familia.

\section{Resultados}

Los parámetros fisicoquímicos muestran cuerpos de agua con características propias de ambientes acuáticos de agua negra, de ligera acidez - a excepción de una de las estaciones-, baja conductividad, temperatura de $28,5^{\circ} \mathrm{C}$ en promedio, transparencia de $0,5 \mathrm{~m}$ y bajo oxígeno disuelto. En la descripción de los hábitats, observamos que las estaciones estuvieron caracterizadas por distintos microhábitat: ambientes lóticos con orillas arenosas, gramalotales y palizadas, así como el ambiente de 'cocha' con sustrato areno-fangoso y fangoso; con diferencias entre épocas (Tabla 1).

Fueron colectados 1626 individuos, correspondientes a 86 especies, pertenecientes a 60 géneros, 21 familias y cinco órdenes, de los cuales los órdenes Characiformes y Siluriformes conforman más del $75 \%$ tanto en número de individuos como en número de especies, géneros y familias (Tabla 2).

Del total de especies identificadas, se registran tres nuevos géneros para la cuenca del rio Nanay, Dysichthys, Pseudoloricaria (Siluriformes) y Laetacara (Perciformes), en total fueron 17 especies registradas por primera vez para la zona. En el Tabla 3 se presenta la lista de especies para la cuenca baja del río Nanay, tanto para época de vaciante como para época de creciente; las familias fueron ordenadas según Reis et al.(2003) y los géneros y especies en orden alfabético dentro de las familias.

Moenkhausia aff. ceros, Curimatella sp. y Hemiodus sp. del orden Characiformes (52,5\%) y Amblydoras nauticus del orden Siluriformes (9\%) conforman el $61,5 \%$ de la abundancia total encontrada. En época de vaciante predomina Amblydoras nauticus, constituyendo el 28,5\% de los individuos en dicha época, mientras que las especies Moenkhausia aff. ceros y Curimatella sp. destacan en época de creciente, con un $65,8 \%$.

El orden con mayor riqueza específica fue Characiformes, con el 66\% del número de especies, siguiendo Siluriformes, con $18 \%$. La familia con el mayor número de especies fue Characidae, representando el 36\%, seguida por Cichlidae (14\%), Curimatidae (10\%), Loricariidae (6\%), para luego dar paso a las otras 18 familias que juntas conforman aproximadamente el $34 \%$.

En cuanto a la abundancia, el orden con el mayor número de individuos fue Characiformes, que obtuvo un $80 \%$ del total

Tabla 2. Número de familias, especies e individuos por órdenes registrados en la cuenca baja del río Nanay.

\begin{tabular}{ccccc}
\hline Orden & Familias & Géneros & Especies & Individuos \\
\hline Characiformes & 12 & 34 & 56 & 1297 \\
Siluriformes & 6 & 13 & 16 & 205 \\
Perciformes & 1 & 11 & 12 & 108 \\
Beloniformes & 1 & 1 & 1 & 3 \\
Clupeiformes & 1 & 1 & 1 & 13 \\
\hline Total & $\mathbf{2 1}$ & $\mathbf{6 0}$ & $\mathbf{8 6}$ & $\mathbf{1 6 2 6}$ \\
\hline
\end{tabular}


Tabla 3. Lista Sistemática y abundancia total de las especies de peces de la cuenca baja del río Nanay por estaciones y épocas (V: Vaciante, Cr: Creciente; C: Consumo, O: Ornamental, OP: Con Potencial Ornamental, NR: Nuevos Registros).

\begin{tabular}{|c|c|c|c|c|c|c|c|c|c|c|c|c|}
\hline \multirow[t]{2}{*}{ OrdenFamilia } & \multirow[t]{2}{*}{ Especies } & \multicolumn{2}{|c|}{ Pampachica } & Las $\mathrm{C}$ & nelias & Pto. A & endras & Nin & Rumi & Total & Usos & NR \\
\hline & & $\mathbf{V}$ & $\mathrm{Cr}$ & $\mathbf{V}$ & $\mathrm{Cr}$ & V & $\mathrm{Cr}$ & $\mathrm{V}$ & $\mathrm{Cr}$ & & & \\
\hline Characiformes & & & & & & & & & & & & \\
\hline Curimatidae & Curimata vittata & & & & & 6 & & 3 & & 9 & $\mathrm{O}-\mathrm{C}$ & \\
\hline & Curimatella meyeri & & & 21 & & & & & & 21 & $\mathrm{OP}$ & $x$ \\
\hline & Curimatella sp. & & 5 & 9 & 2 & 2 & 192 & & 120 & 330 & $\mathrm{O}$ & \\
\hline & Curimatopsis macrolepis & & & & & & & & 1 & 1 & $\mathrm{O}-\mathrm{C}$ & \\
\hline & Cyphocharax spilurus & & & & 6 & & 14 & & 16 & 36 & OP & \\
\hline & Psectrogaster sp. & & & & 5 & & & & & 5 & $\mathrm{C}$ & \\
\hline & Psectrogaster essequibensis & & & & 4 & & 6 & & 5 & 15 & $\mathrm{C}$ & $x$ \\
\hline & Steindachanerina aff. guentheri & & & & & & & & 5 & 5 & OP & \\
\hline & Steindachnerina sp. & & & & & & & & 15 & 15 & C & \\
\hline Prochilodontidae & Prochilodus nigricans & 3 & & & & & & & & 3 & $\mathrm{O}-\mathrm{C}$ & \\
\hline & Semaprochilodus theraponura & & & 12 & & & & & & 12 & $\mathrm{O}-\mathrm{C}$ & \\
\hline Anostomidae & Anostomus sp. & & & & & 6 & & & & 6 & $\mathrm{O}$ & \\
\hline & Leporinus agassizi & & 4 & & & & & & & 4 & $\mathrm{O}-\mathrm{C}$ & \\
\hline & Leporinus friderici & & & & 1 & & 1 & & 1 & 3 & $\mathrm{O}-\mathrm{C}$ & \\
\hline Chilodontidae & Chilodus punctatus & & & 5 & 1 & & 1 & & 1 & 5 & $\mathrm{O}$ & \\
\hline & Chilodus sp. & & & 5 & & & & & 1 & 6 & $\mathrm{OP}$ & \\
\hline Crenuchidae & Characidum etheostoma & & & & & & 9 & & 2 & 11 & $\mathrm{O}$ & \\
\hline Hemiodontidae & Hemiodus sp. & 42 & 13 & 4 & 32 & 7 & & 2 & & 100 & $\mathrm{C}$ & \\
\hline Gasteropelecidae & Carnegiella myersi & & & 2 & & & & & & 2 & $\mathrm{O}$ & \\
\hline Characidae & Astyanax bimaculatus & & 2 & & & & & & & 2 & $\mathrm{O}$ & \\
\hline & Brycon pesu & 1 & & & & & & & & 1 & $\mathrm{O}$ & \\
\hline & Bryconops melanurus & & & & & 4 & & & & 4 & C & \\
\hline & $\begin{array}{l}\text { Bryconops sp. } \\
\text { B. }\end{array}$ & & & & & 4 & 2 & & & $\begin{array}{l}4 \\
2\end{array}$ & $\mathrm{O}-\mathrm{C}$ & \\
\hline & Chalceus sp. & & 1 & & & 1 & 2 & & 2 & 6 & $\mathrm{O}$ & \\
\hline & Cheirodon ortegai & & 1 & & & 1 & 3 & & 2 & 3 & OP & $x$ \\
\hline & Ctenobrycon sp. & & & 1 & 10 & & & & & 11 & $\mathrm{O}$ & \\
\hline & Hemigrammus hyanuari & & & 1 & 10 & & 8 & & & $\begin{array}{c}11 \\
8\end{array}$ & $\mathrm{O}$ & \\
\hline & Hemigrammus levis & & & & & & 30 & & & 30 & $\mathrm{O}$ & \\
\hline & Hemigrammus pulcher & & & 1 & & & $\begin{array}{c}50 \\
7\end{array}$ & & 2 & 10 & $\mathrm{O}$ & \\
\hline & Iguanodectes spilurus & & & & & 1 & & & & 1 & $\mathrm{O}-\mathrm{C}$ & \\
\hline & Knodus sp. & & 9 & & & & & & & 9 & $\mathrm{O}$ & \\
\hline & Moenkhausia aff. ceros & & 70 & 10 & 2 & & 212 & & 130 & 424 & $\mathrm{O}$ & \\
\hline & Moenkhausia aff. collettii & & & 7 & & & & & & 7 & $\mathrm{O}$ & \\
\hline & Moenkhausia aff. comma & & & 3 & & 4 & & 7 & & 14 & $\mathrm{O}$ & \\
\hline & Moenkhausia aff. cotinho & & 1 & & 1 & & 6 & & 5 & 13 & $\mathrm{O}$ & \\
\hline & Moenkhausia aff. dichroura & & & & & & 34 & & & 34 & $\mathrm{O}$ & \\
\hline & Moenkhausia aff. intermedia & & & 1 & 1 & & & & & 2 & $\mathrm{O}$ & \\
\hline & Moenkhausia chrysargyrea & 3 & & & & & & & & 3 & $\mathrm{O}$ & \\
\hline & Moenkhausia colletti & & & & 2 & & & & & 2 & $\mathrm{O}$ & \\
\hline & Moenkhausia dichroura & & & 2 & & & & & & 2 & $\mathrm{O}$ & \\
\hline & Moenkhausia intermedia & & & 2 & & & 2 & & & 4 & $\mathrm{O}$ & \\
\hline & Moenkhausia jamesi & & & & 1 & & 1 & & & 2 & $\mathrm{O}$ & $x$ \\
\hline & Moenkhausia lepidura & 3 & 3 & 14 & & & 6 & 3 & & 29 & $\mathrm{O}$ & \\
\hline & Moenkhausia oligolepis & & & & & & 3 & & 1 & 4 & $\mathrm{O}$ & \\
\hline & Moenkhausia sp. & 39 & & & & & & & & 39 & $\mathrm{O}$ & \\
\hline & Myleus rubripinnis & & & & & 4 & & & & 4 & $\mathrm{O}-\mathrm{C}$ & \\
\hline & Serrasalmus aff. rhombeus & & & & & & & & 3 & 3 & $\mathrm{O}-\mathrm{C}$ & \\
\hline & Serrasalmusaff. nattereri & & & 1 & & & & & & 1 & $\mathrm{O}-\mathrm{C}$ & \\
\hline & Tetragonopterus argenteus & 1 & & & & & & & & 1 & $\mathrm{O}$ & \\
\hline & Triportheus elongatus & & & & & 1 & & & & 1 & $\mathrm{O}$ & \\
\hline Acestrorhynchidae & Acestrorhynchus falcirostris & & 2 & 1 & & & & & & 3 & $\mathrm{O}-\mathrm{C}$ & \\
\hline Erythrinidae & Hoplerythrinus unitaeniatus & & 1 & & & & & & & 1 & $\mathrm{O}-\mathrm{C}$ & \\
\hline & Hoplias malabaricus & 9 & 8 & & 1 & 1 & & 1 & & 20 & $\mathrm{C}$ & \\
\hline Lebiasinidae & Pyrrhulina brevis & & & & 1 & & & & & 1 & $\mathrm{O}$ & \\
\hline Ctenolucidae & Boulengerella cuvieri & & 1 & & 1 & & 1 & & 1 & 4 & $\mathrm{O}$ & $x$ \\
\hline & Boulengerella maculata & & & & & 1 & 1 & & 1 & 3 & OP & \\
\hline Siluriformes & & & & & & & & & & & & \\
\hline Aspredinidae & Dysichthys sp. & 1 & & & & & & & & 1 & $\mathrm{OP}$ & $\mathrm{X}$ \\
\hline Trichomycteridae & Ochmacanthus sp. & & & & & & & 1 & & 1 & $\mathrm{OP}$ & $x$ \\
\hline Callichthyidae & Megalechis thoracata & & & 1 & & & & & & 1 & OP & $x$ \\
\hline Loricariidae & Limatulichthys punctatus & 11 & & 1 & & & & & & 11 & OP & $\hat{X}$ \\
\hline & Limatulichthys sp. & & & & & 13 & & & & 13 & OP & $x$ \\
\hline & $\begin{array}{l}\text { Oxyropsis sp. } \\
\text { Oxp. }\end{array}$ & & & & & & 2 & & 2 & $\begin{array}{c}13 \\
4\end{array}$ & OP & $\hat{x}$ \\
\hline & Pseudoloricaria sp. & & & & & 2 & & & & 2 & OP & $x$ \\
\hline & Rineloricaria morrowi & 2 & & & & 2 & & & & 4 & OP & \\
\hline Heptapteridae & Pimelodella cristata & & & & & 6 & & & & 6 & OP & $x$ \\
\hline перtapterruae & Pimelodella gracilis & 1 & & & & & & & & $\begin{array}{l}0 \\
1\end{array}$ & OP & 入 \\
\hline Pimelodidae & Goeldiella aff. eques & 1 & & & & & & & & 1 & $\mathrm{O}$ & \\
\hline Pmerourade & Pimelodus maculatus & & & & & 1 & & & & $\begin{array}{l}1 \\
1\end{array}$ & $\mathrm{O}$ & $x$ \\
\hline & Pimelodus tetramerus & & & & & 1 & & 3 & & & $\mathrm{O}-\mathrm{C}$ & $\mathrm{X}$ \\
\hline Doradidae & Amblydoras nauticus & 4 & & 142 & & & & & & $\begin{array}{c}4 \\
146\end{array}$ & $\mathrm{O}$ & \\
\hline & Nemadoras trimgculatus & 1 & & & & & & & & 1 & $\mathrm{OP}$ & \\
\hline & Physopixys lyra & & & 4 & & & & 4 & & $\begin{array}{l}1 \\
8\end{array}$ & $\mathrm{O}$ & \\
\hline Perciformes & & & & & & & & & & & & \\
\hline Cichlidae & Acaronia nassa & & 7 & & 1 & & 6 & 5 & & 19 & $\mathrm{O}$ & $X$ \\
\hline & Aequidens sp. & & & & & & & 16 & 1 & 17 & $\mathrm{O}-\mathrm{C}$ & \\
\hline
\end{tabular}


Tabla 3.

\begin{tabular}{|c|c|c|c|c|c|c|c|c|c|c|c|c|}
\hline & $\begin{array}{l}\text { Aequidens tetramerus } \\
\text { Apistogramma luelingi } \\
\text { Biotodoma cupido } \\
\text { Cichla monoculus } \\
\text { Cichlasoma amazonarum } \\
\text { Crenicichla anthurus } \\
\text { Heros apendiculatus } \\
\text { Laetacara thayeri } \\
\text { Mesonauta mirificus } \\
\text { Satanoperca jurupari }\end{array}$ & $\begin{array}{l}6 \\
3 \\
2 \\
2\end{array}$ & $\begin{array}{l}6 \\
6 \\
\\
2 \\
2 \\
1 \\
\\
3\end{array}$ & & 1 & 1 & $\begin{array}{l}2 \\
3 \\
8 \\
\end{array}$ & $\begin{array}{l}7 \\
1 \\
\end{array}$ & $\begin{array}{l}1 \\
7 \\
\end{array}$ & $\begin{array}{c}13 \\
6 \\
3 \\
2 \\
4 \\
4 \\
1 \\
2 \\
11 \\
26 \\
\end{array}$ & $\begin{array}{l}\mathrm{O}-\mathrm{C} \\
\mathrm{OP} \\
\mathrm{O} \\
\mathrm{O}-\mathrm{C} \\
\mathrm{O} \\
\mathrm{O} \\
\mathrm{O} \\
\mathrm{OP} \\
\mathrm{O} \\
\mathrm{O}-\mathrm{C}\end{array}$ & $X$ \\
\hline \multicolumn{13}{|l|}{ Beloniformes } \\
\hline $\begin{array}{c}\text { Belonidae } \\
\end{array}$ & Potamorrhaphis sp. & 3 & & & & & & & & 3 & $\mathrm{O}$ & \\
\hline $\begin{array}{c}\text { Clupeiformes } \\
\text { Engraulididae }\end{array}$ & Anchoviella sp. & & & & & 1 & 7 & 1 & 4 & 13 & C & \\
\hline 21 & 86 & 138 & 147 & 248 & 73 & 72 & 568 & 54 & 326 & 1626 & & 17 \\
\hline
\end{tabular}

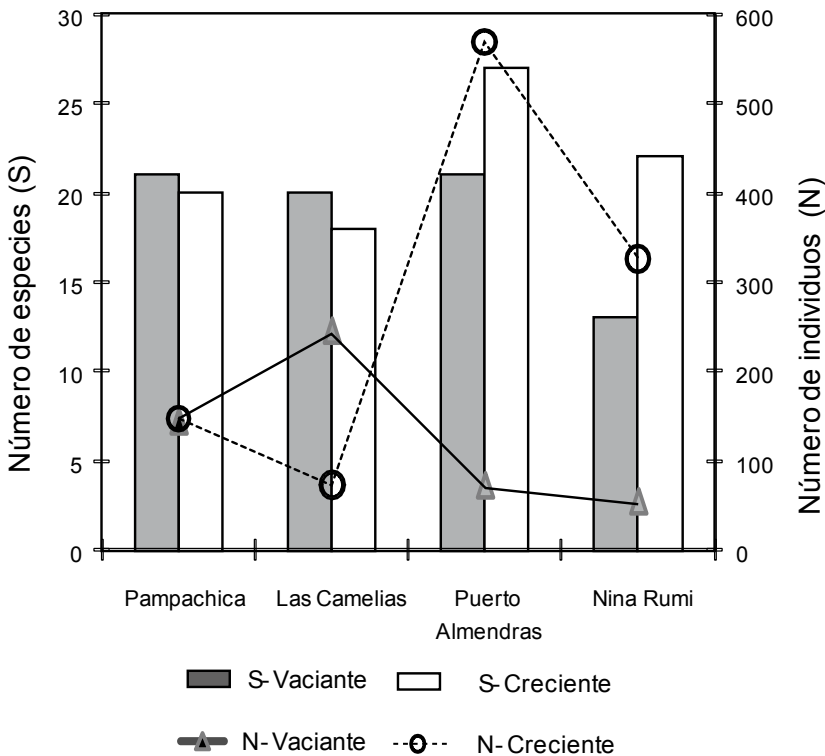

Figura 1. Riqueza (S) y abundancia (N) de peces en la cuenca baja del río Nanay.

de individuos colectados; seguido de Siluriformes con un 13\%, Perciformes con el 7\%, Clupeiformes con el 1\% y finalmente Beloniformes. Characidae fue también la familia con el mayor porcentaje en el número de individuos ( $42 \%$ del total).

En época de vaciante se capturaron 512 individuos pertenecientes a 58 especies, 45 géneros, 19 familias y cinco órdenes, mientras que en época de creciente fueron colectados 1114 ejemplares, identificándose 48 especies, 34 géneros, 13 familias y cuatro órdenes. En época de vaciante, los órdenes Characiformes, y Siluriformes fueron los predominantes en cuanto a riqueza de especies, mientras que en creciente los Characiformes y Perciformes fueron los ordenes resaltantes, con 31 y 11 especies. En relación al número de individuos, los Characiformes también fueron los que tuvieron los mayores valores, con 256 individuos en época de vaciante y 1041 en época de creciente. Las familias Characidae y Cichlidae fueron las mejor representadas en época de vaciante; mientras que Characidae y Cichlidae presentaron los mayores valores en número de especies. Doradidae presento más individuos en época de vaciante, y Characidae en época de creciente. Variantes con respecto a riqueza y abundancia entre épocas y estaciones las podemos observar en la Figura 1.

El dendrograma en la Figura 2 reúne a todas las estaciones en todas las épocas, en el que podemos dar cuenta de la formación de un grupo claro de las estaciones en Pampachica, Puerto Almendras y Nina Rumi en época de vaciante y Las Camelias en época de creciente. La estación en la localidad de Pampachica en época de creciente tendría similitud con el grupo formado anteriormente. Se observó similitud entre las estaciones de Nina Rumi y Puerto Almendras en época de creciente, y hallamos que la más diferente entre todas las locaciones fue la estación en Las Camelias, en época de creciente.

\section{Discusión}

En general se confirma la predominancia de Characiformes y Siluriformes (Super Orden Ostariophysi en la cuenca baja del río Nanay), la cual sigue el patrón perteneciente a las aguas con-

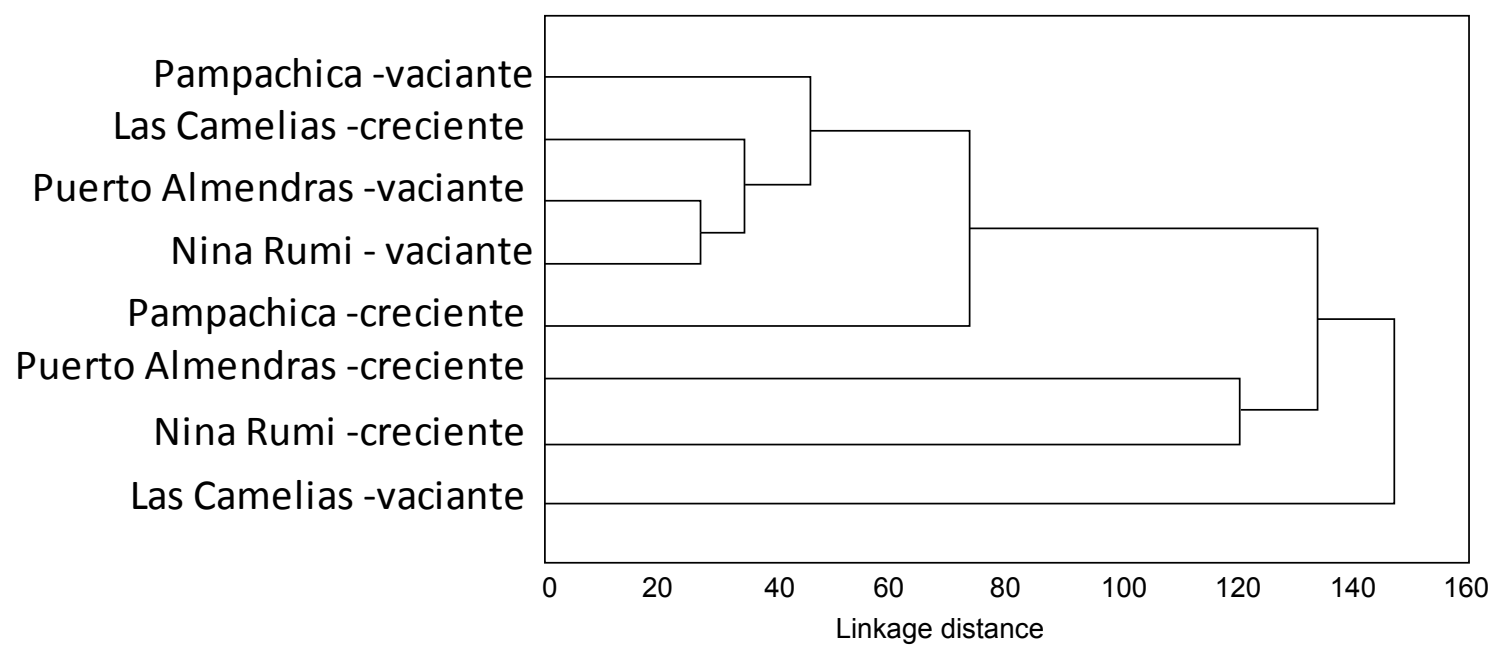

Figura 2. Dendrograma de los grupos formados por todas las estaciones de muestreo en ambas épocas (Distancia Euclidiana). Cuenca baja del río Nanay (2007-2008). 
tinentales de la Región Neotropical (Lowe-McConnell, 1987). Las familias Characidae y Cichlidae son las predominantes en riqueza y conforman el 50\% de las especies. Estos resultados coinciden con aquellos publicados por el IIAP (2002). La predominancia de carácidos puede ser explicada por la variedad de microhábitat encontrados en el río Nanay; los hábitos alimenticios y los diferentes tipos de dientes entre las especies y sus diversos hábitos reproductivos estarían adecuados a dicha variedad de microhábitat. La presencia de varias especies de cíclidos se debería a sus hábitos reproductivos, su territorialismo y cripticismo -importante en agua negra.

La lista de especies (Tabla 3) actualiza la información sobre los peces de la cuenca baja del río Nanay. Así, el 27,9\% de las especies identificadas fueron nuevos registros y el 8,14\% del total de las especies fueron reportadas por primera vez, es decir el $19,8 \%$ de las especies identificadas en el presente trabajo fueron nuevos registros.

La estructura comunitaria está basada en especies de porte pequeño $(<10 \mathrm{~cm})$, representando el $61,5 \%$ de los individuos, en su mayoría Characiformes; esto puede deberse a que dichas especies tienen una relación más estrecha con las orillas, remansos de los ríos y los ambientes de baja velocidad creados en época de creciente, en los que también se forman diversos microhábitat. Resultados parecidos obtuvo el IIAP (2002).

La representatividad de familias Characiformes en abundancia, se explicaría por la variedad y disponibilidad de alimento, como al material en semidescomposición, propio de ríos de agua negra, encontrado en el fondo del río sobre el material arenoso; además de los hábitos de formación de asociaciones entre las especies de dichas familias (Géry 1977).

El mayor número de individuos en época de creciente (1114), podría responder a la formación de ambientes lénticos temporales y a las planicies inundables donde se albergan juveniles de muchas especies (Géry 1977).

El dendrograma muestra que la estación de Las Camelias, en época de creciente, es similar a las otras tres estaciones en época de vaciante, esto podría ser explicado por el aporte y al intercambio de especies existente entre el río y el cuerpo léntico existente en época de creciente; la composición, estructura, y abundancia relativa de especies fundamentan dicho agrupamiento. Por otro lado durante la época de creciente las diferencias en composición y estructura se hacen más notables entre los lugares estudiados.

El índice de abundancia (41 individuos/lance en promedio), obedece tanto a características etológicas de los peces tales como el gregarismo (Géry 1977), así como a factores ambientales tales como el ciclo hidrológico. Así, podemos ver que en las estaciones con un mayor número de especies Characiformes, especialmente de las familias Characidae, Curimatidae y Hemiodontidae, son aquellas en las que la abundancia fue mayor, lo que se debería a sus hábitos gregarios. Se encontraron diferencias entre épocas, siendo la época de creciente la que muestra una mayor abundancia relativa tanto en sumatoria como en promedio.

La estación de Las Camelias presento la diversidad más baja en la época de creciente, lo que podría atribuirse principalmente a la intensa pesca de peces de uso ornamental, en especial de Symphysodon aequifasciatus, actividad que se realiza tanto en época de vaciante como en época de creciente.
La captura de peces de uso ornamental se realiza en toda la cuenca baja y media del río Nanay, especialmente desde aquellos lugares cercanos a la ciudad de Iquitos, con acceso desde la carretera Iquitos-Nauta. La captura se realiza con malla mosquitera, de 1 a $3 \mathrm{~mm}$ de abertura; capturando indiscriminadamente peces ornamentales y juveniles de peces de consumo. Esta captura es realizada de pequeña a mediana escala por las empresas acuaristas e informalmente por pobladores de caseríos asentados a orillas del río. El proceso de comercialización fue descrito en su totalidad por Tello y Cánepa (1991). En la Tabla 3 podemos observar que la mayoría de las especies tienen uso ornamental (56 especies solo ornamentales, indicados con $\mathrm{O}$ en la lista, y 19 especies tanto ornamentales como de consumo, representadas por $\mathrm{O}-\mathrm{C}$ ). También se identificaron peces con potencial ornamental (OP), que reúnen características tales como el porte pequeńo, colores brillantes o vistosos, comportamiento o hábitos especiales y fáciles de criar en espacios pequeños. Gran parte de las familias, en este estudio, incluyen a peces ornamentales, siendo solo tres aquellas que no incluyen a este tipo de peces (Tabla 3).

\section{Agradecimientos}

A los Doctores Mark Sabaj (Academia de Ciencias Naturales de Filadelfia) y Flavio Lima (Museo de Zoología de la Universidad de Sao Paulo) por su ayuda en la identificación de especies de las familias Doradidae y Characidae, respectivamente. Al Departamento de Ictiología del Museo de Historia Natural de la Universidad Nacional Mayor de San Marcos por el ambiente y equipos prestados.

\section{Literatura citada}

Castello L. 2007. Lateral migration of Arapaima gigas in floodplains of the Amazon. Ecology of freshwater fish. Singapur. 1-9 pp.

Chang F. \& H. Ortega. 1995. Additions and Corrections to the List of Freshwater Fishes of Peru. Publ. Mus. Hist. Nat. UNMSM (A) 50:1-12.

Gerstner C., H. Ortega, W. Graham \& H. Sánchez. 2006. Effects of the freshwater aquarium trade on wild fish populations in differentially-fished areas of the Peruvian Amazon. Journal of Fish Biology. U.S.A. 68 (3): p. 862-875.

Géry J. 1977. Characoids of the World. TFH Publications. Neptune City, New Jersey. U.S.A. 672 pp.

Hidalgo M. \&. P. Willink. 2007. Peces/Fishes. In: Vriesendorp et. al. eds. Perú: Nanay-Mazan-Arabela. Rapid Biological Inventories Report 18. The Field Museum. Chicago, U.S.A. 56-62 pp.

IIAP (Instituto de Investigaciones de la Amazonia Peruana). 2002. Propuesta de Zonificación Ecológica Económica de la Cuenca del Río Nanay. Programa de Ordenamiento Ambiental. IIAP. Iquitos, Perú. Vol. I-IV.

Kahn F.; B. León \& K.R. Young (Compiladores). 1993. Las plantas vasculares en las aguas continentales del Perú. IFEA. Lima-Perú. 357 pp.

Lowe-Mcconnell R.S. 1987. Ecological Studies in Tropical Fish Communities. New York. Cambridge University Press. $381 \mathrm{pp}$.

Manly B. F.J. 1985. Multivariate Statistical Methods, A primer. Chapman and Hall. U.S.A. pp. 1-159.

Ortega H. 2007. Composición, Distribución y Conservación de la Comunidad de Peces en la Zona Reservada del Parque Nacional Manu. Universidad Nacional Mayor de San Marcos. Tesis para obtener el Grado Académico de Magíster en Zoología. Lima, Perú. 1-99 pp. 
Ortega H., \& R.P. Vari. 1986. Annotated Checklist of the Freshwater Fishes of Peru. Smithsonian Contributions to Zoology 437. Washington, D.C. USA. 1-25 pp.

Ortega H. \& E. Castro. 1998. Hidrobiología de la Cuenca del Río Nanay. Evaluación de la Ictiofauna, Diagnostico de la Pesquería y Propuesta de Manejo de los Recursos Hidrobiológicos. CEDIA. Lima, Perú. 60pp.

Roldán G. 1992. Fundamentos de Limnología Neotropical. Editorial Universidad de Antioquía. Colombia. 529 pp.

Reis R.E., S.O. Kullander \& C.J. Ferrari Jr. 2003. Check list of the freshwater fishes of South and Central America. EDPUCRS, Porto Alegre, Brasil. 729 pp.

Rico-G.A., J. Beltrán, A. Álvarez \& E. Flores. 2005. Diversidad de arañas (Arachnida: Araneae) en el Parque Nacional Natural Isla Gorgona, Pacífico Colombiano. Biota Neotrop. [online]. 5(1a): 99-110 . Disponible en: <http://www. scielo.br/scielo.php?script $=$ sci arttext\&pid $=\mathrm{S} 1676$ $06032005000200009 \& \operatorname{lng}=\mathrm{es} \& \mathrm{nrm}=\mathrm{iso}>$. (acceso: $02 / 11 / 2009)$
Sioli H. 1984. The Amazon, Limnology and landscape ecology of a mighty river and its basin. Dr. W. Junk Publishers. Dordrecht Netherlands and Boston and Hingham, MA, USA. 763 pp.

Souza J., A. Frias, U. Vela \& L. Verdi. 2004. Principales hábitats para la captura de peces ornamentales en los ríos Nanay y Ucayali. Memorias: VI Congreso Internacional de Manejo de Fauna silvestre en Amazonia y Latinoamérica. Iquitos. Perú. 296-299pp.

Tello S. \& J. Cánepa. 1991. Estado actual de la explotación de los principales peces ornamentales de la Amazonía Peruana. Folia Amazónica IIAP. Perú. Vol. No3. 107-128pp. 